\title{
Research on Electronic Educational Administration Platform
}

\author{
Tao $\mathrm{Qu}^{1, \mathrm{a}}{ }^{*}$ and Jinyu $\mathrm{Lu}^{2, \mathrm{~b}}$ \\ ${ }^{1}$ School of Electronics \& Information Engineering University of Technology Liaoning Liaoning, \\ Jinzhou, China \\ ${ }^{2}$ College of Engineering Bohai University Liaoning, Jinzhou, China \\ a54333928@qq.com, b156241142@qq.com
}

\begin{abstract}
Keywords: Web Service; Electronic educational administration platform; Systematic structure; Expansion SOAP
\end{abstract}

\begin{abstract}
In this paper, the author analyzes the current situation of electronic educational platform, introduces the systematic structure of the Web Service and the service agreement. And the author from two aspects of special interfaces and expansion SOAP [1], put forward design scheme of electronic educational administration platform based on Web Service, gave the design program of electronic educational administration platform, especially focused on the key part of the program.
\end{abstract}

\section{Introduction}

With the continuous expansion of the scale of the university, the improvement of educational quality level and an increasing number of workers the departments of university used to develop an information management system to meet their actual situation and demands. But the information management systems are different in platform of hardware and software. In technology standard, and be developed by different software, and they are "information isolated islands". Therefore, it is urgent problems to be solved that how to integrate the information isolated islands. The establishment of electronic educational administration platform is imperative. Electronic educational administration platform is based on the digital information, take the computer technology and the network system as the basis, realization of University Teaching Science, scientific research, management and service of campus information collection, processing, Integration, storage, transmission and application, make the teaching resources used fully.

Web Service provides a solution to build mutual application interface and data services interface between different system architectures. Web Service hides the details of the service, so hardware platform, software platform and programming language is independent, which also makes applications based on Web Service with loosely coupled, component-based and cross- platform features. Web Service that apply SOAP, UDDI[2-4], WSDL[5-8] and HTTP technology integrate information isolated islands, form a distributed, automated and intelligent network applications, it improve the efficiency of program development, and full use of the network resources.

\section{Web Service Architecture Technology}

Web Service based on XML system framework and distribute computing standard, using the unified method of service description, register and query. Web Service provides an open, efficient and interoperable framework for the operation.

System Structure of the Web Service. Web Service architecture is based on the interaction between three kinds of roles (service provider, service registry and service requestor).

Interactions involve release, find and binding operations. The roles and operations act on the member of Web Services together. The service provider defines Services Web Service description. And publish it to the service requester or Service registry. Service requestor using a find operation to search service description from the local or Service registry, and then use the service description 
binding Service provider. And call the Web Service implementation or interactions. As shown in Fig. 1 .

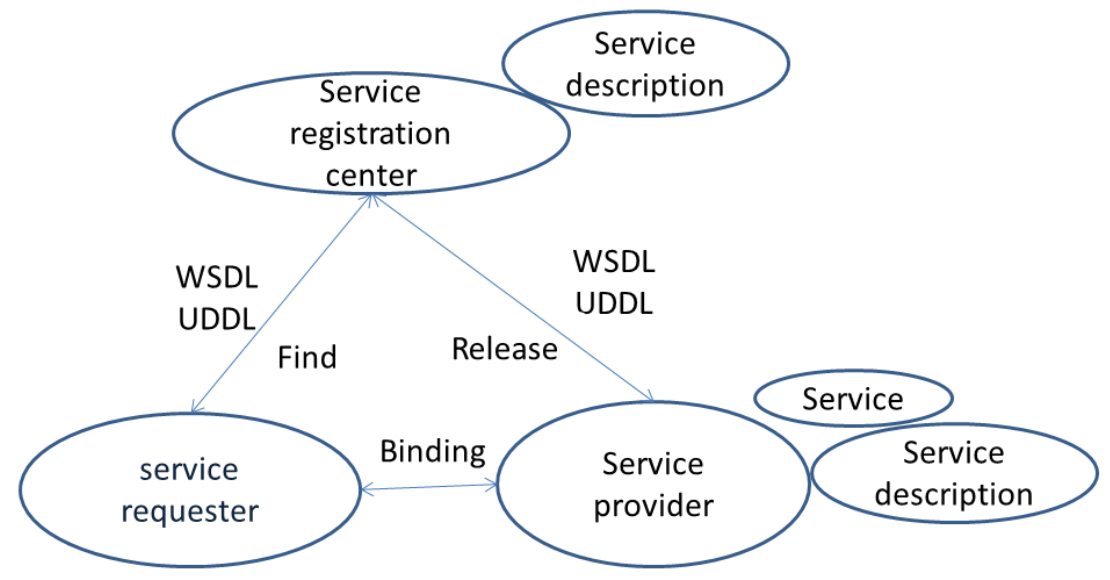

Figure 1. System structure of the Web Service

Service Protocol of Web Services. SOAP is simple information protocol, which is based on XML protocol. SOAP did not define application semantics, but it format information with the XML, carrying messages by http. The http protocol is internet protocol. The HTTP protocol can be used to invoke remote COM object to across the network and firewall restrictions. SOAP protocol involves the SOAP envelope, coding rules and RPC represent $\mathrm{t}$ [9-11].

WSDL Web Services need to be some kind of structured way to describe its own function and its invocation and communication that is the only way, Web Services can be invoked by a detailed understanding. A WSDL service description contains an abstract definition of a set of actions and messages, to bind to a specific protocol and message of these operations and the binding of a network endpoint specification.

UDDI is a standard specification which based wed, distributed, and provided information to registry for Web Service. It provides a method for publishing and searching service description. Depending on Standard interface provided by UDDI, Enterprises or individuals may issue the Web Service, for the enterprise or the individual query, also can let other companies or individuals to access Web Service services.

\section{Design of Electronic Educational Administration Platform Based on Web Service}

Web Service is based on XML format, using the loose coupling way, heterogeneous application systems[9] can be connected through a dedicated interface, so it has the characteristics of universality, interoperability, easy to use and high scalability, is the best choice for connecting heterogeneous application systems. The author proposes a university electronic educational administration platform scheme based on Web Service (as shown in "Fig. 2").

Special Interface. The special interface is the bridge of heterogeneous systems to exchange information with SOAP. Design the special interface includes two aspects: one is the different system structures of different interface. Only in this way, heterogeneous system can call Web Service platform; two is the data transformation services, for heterogeneous system sends the message, the data transformation services verify the validity and generate WSDL. And implementation of data format conversion between heterogeneous systems and SOAP.

Extended SOAP. The communication process between the client and the Web Service mainly has 9 parts: the client code to create a proxy object and call its methods: the actual call of the proxy was serialized as an SOAP request message; SOAP request message was transmitted to the server through Internet: the SOAP request message was desterilized to the method call; the server invoke the corresponding Web Service object: the server serialized the result of a method call as an SOAP response message: SOAP sent messages to the client through the Internet: the client desterilized the 
SOAP response message and passed it to the proxy object; proxy object returns the results back to the client. Among them, SOAP is the key part of Web Service services. In the Web Service application, the client calls the Web Service method, the SOAP response may be large data sets in the transmission network. Especially in the database, SOAP response may contain large amounts of data, may be tens of thousands of rows sometimes. For the data set, it can affect the network bandwidth seriously during transmission, must be controlled. Therefore, prior to data transmission, we compressed transmission data, in order to reduce the data flow, improve the transmission efficiency.

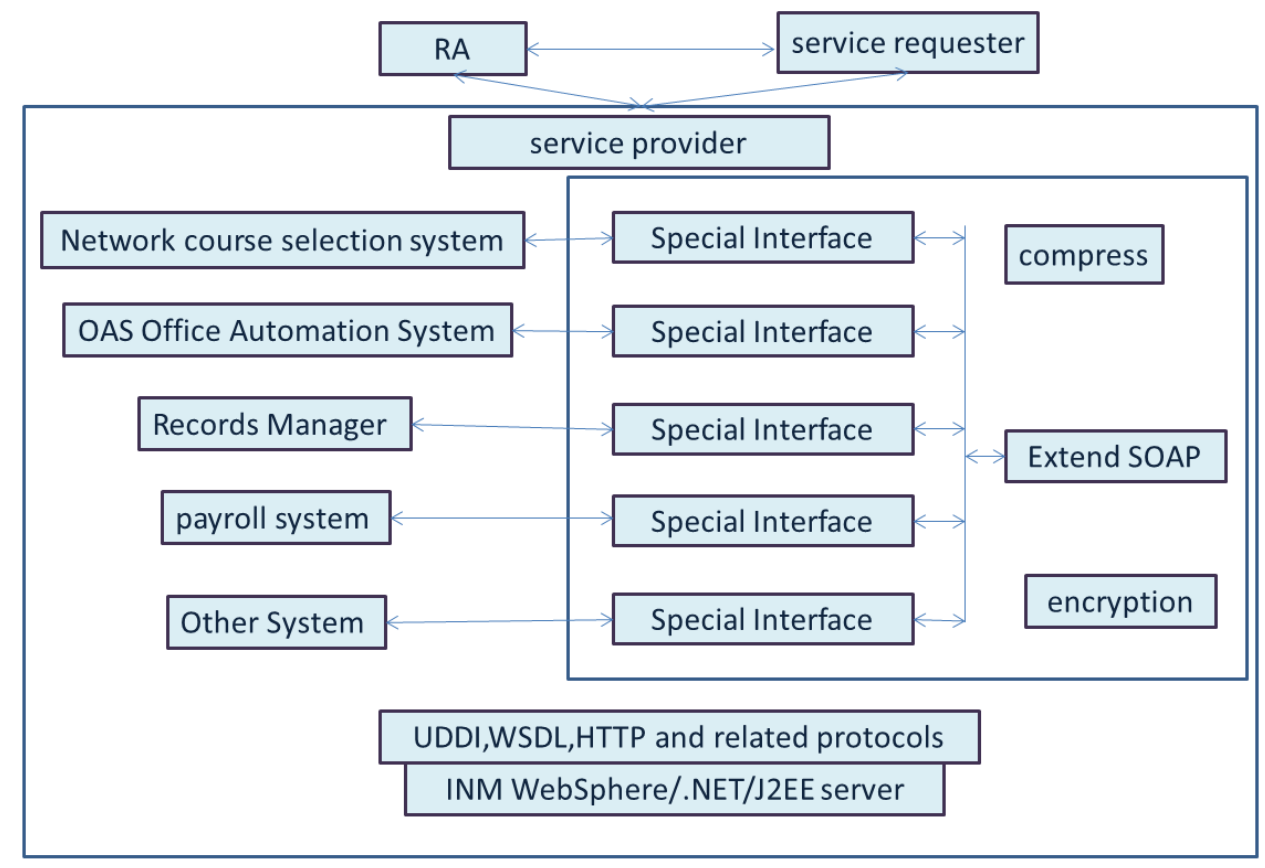

Figure 2. Design scheme of electronic educational administration platform

In order to reduce the data flow, should compress the data before sending the SOAP data to serialize, and extract the data before the parallel of the SOAP data.

When the SOAP message transmission in HTTP, in order to improve the security of data transmission, the transmission should be encrypted content, while the data transport to the client, and then decrypts the data. Encryption and decryption can used DES algorithm which is open. You can also use the asymmetric encryption algorithm, namely the client to use the server's public key to encrypt the SOAP message, and the server using a private key to decrypt the encrypted data.

\section{Conclusions}

Based on Web Service characteristics, the author has conducted the research to the key part of the University of Electronic Educational Administration Platform based on the Web Service. Web Service is a service-oriented architecture, which is loose coupling, interoperability, universality, and easy to use, it has been widely applied in business. At present, the Microsoft and IBM support the DUUI service, and put forward a series of technical specification, which will promote the further development and improvement of Web Service.

\section{References}

[1] Yu Pingxiang,Wu Yuanmei:Transactions of the Chinese Society of Agricultural Engineering,2008,24(7):132-136 
[2] S. Yin, X. Li, H. Gao, O. Kaynak, Data-based techniques focused on modern industry: An overview,IEEE Transactions on Industrial Electronics,62(1):657-667, 2015.

[3] LI Mengxun,Liu Hongzhi: Journal of Food Science and Technology ,2011,29(2),P54-58

[4] S. Yin, Z. Huang, Performance monitoring for vehicle suspension system via fuzzy positivistic C-means clustering based on accelerometer measurements, IEEE/ASME Transactions on Mechatronics, 20(5):2613-2620, 2015.

[5] S. Yin, X. Zhu, O. Kaynak, Improved PLS focused on key performance indictor related fault diagnosis, IEEE Transactions on Industrial Electronics, 62(3):1651-1658, 2015.

[6] Yu Pingxiang, $\mathrm{Wu}$ Yuanmei: Transactions of the Chinese Society of Agricultural Engineering,2008,24(7)p132-136

[7] S. Yin, O. Kaynak, Big data for modern industry: challenges and trends, Proceedings of the IEEE, 102(3):143-146, 2015.

[8] Sun Qibo,Liu Jie: Journal of Beijing University of Posts and Telecommunica-tions, 2010,33(3)p1-9

[9] Dun Wentao, Xia Bin: Modern Agricultural Science and Technology,2012(24)p216-221.

[10] WEI Xiao-xiao Journal of Anhui Agricultural Sciences ,2011,39(24):15150-15152

[11] S. Yin, X. Zhu,?Intelligent particle filter and its application on fault detection of nonlinear system, IEEE Transactions on Industrial Electronics, 62(6):3852-3861, 2015 